\title{
¿CÓMO AFECTA LA CONCIENCIA ECOLÓGICA DEL INDIVIDUO AL MOMENTO DE LA COMPRA DE BIENES DE CONSUMO? UN ESTUDIO APLICADO A CHILE
}

Hidalgo-Alcázar, Carmen

Cofré Tapia, Janett

Cortés Castillo, Macarena

Hurtado Rojas, Inoska

Universidad Catolica del Norte, Chile

mchidalgo@ucn.c

I Material original autorizado para su primera publicación en la revista académica

REDMARKA. Revista Digital de Marketing Aplicado.

https://doi.org/10.17979/redma.2017.01.018.4858

Recibido: 12 Mayo 2017

Aceptado: 10 Junio 2017

\section{Resumen}

A nivel mundial, el planeta se ha ido deteriorando debido a las aceleradas actividades del hombre. Es por esto, que se ha generado un despertar de la conciencia de las personas, adaptando comportamientos amigables con el medio ambiente. Por tanto, un tema que ha tomado gran fuerza en la actualidad es la preocupación del consumidor por el medio ambiente, obligando a las empresas a implementar estrategias de marketing ecológico que busquen satisfacer las necesidades de los clientes y generar el mínimo impacto negativo en el ecosistema. 
La presente investigación tiene por objetivo analizar la conciencia ecológica que presentan los consumidores al momento de realizar la compra en Chile, con el fin de proponer estrategias aplicables al Marketing Ecológico en bienes de consumo. Para concretar el objetivo general, se recogieron datos por medio de una encuesta presencial para determinar el grado de conciencia ecológica y cómo influye esto en la decisión de compra de bienes de consumo.

Los resultados de esta investigación sirven para proponer planes de acción para mejorar la imagen verde de las empresas y para crear o potenciar la conciencia ecológica de los consumidores. Es por ello, que la información entregada en esta investigación en conjunto con los planes de acción propuestos, pretenden contribuir al desarrollo de la sustentabilidad de las empresas y aumentar la concientización de la población.

Palabras clave: marketing ecológico, conciencia ecologica, productos ecológicos, eco-etiquetas, Chile. 


\section{Abstract}

Around the world, the planet has been deteriorating due to the accelerated activities of man. It is because of it, that there has been generated an awakening of the conscience of the persons, adapting friendly behaviors with the environment. Therefore, an issue that has taken great strength at present is the concern of the consumer for the environment, forcing companies to implement strategies of ecological marketing, seeking to meet the needs of customers and generate the minimum negative impact on the ecosystem.

This research aims to analyze the ecological conscience that consumer have at the time of making the purchase in Chile, in order to propose strategies applicable to Marketing eco-consumer goods. To achieve the overall objective, data were collected by means of an in-person survey to determine the degree of ecological consciousness and how it influences the decision to purchase of consumer goods.

The results of this research help to propose plans of action to improve the green image of the companies and to create or improve the environmental consciousness of consumers. Therefore, the information provided in this research with the proposed action plans, they intend to contribute to the development of the sustainability of companies and increasing the consciousness of the population.

Keywords: ecological marketing, ecological consciousness, organic products, ecolabels, Chile. 


\section{INTRODUCCIÓN}

Un tema que ha tomado gran fuerza en la actualidad es la preocupación del consumidor por el medio ambiente, trayendo consigo el nacimiento de diversas estrategias ecológicas por parte de las empresas, con el fin de satisfacer las necesidades de los clientes y generar el mínimo impacto negativo en el ecosistema.

Hoy en día, el cuidado del medio ambiente corresponde, no solo a una tendencia de organizaciones sin fines de lucro, sino que también representa una preocupación para los consumidores de todo el mundo, por lo que las empresas se han visto obligadas a modificar sus hábitos y prácticas para dejar claro que son sustentables.

Existen compañías reconocidas a nivel mundial que aplican Marketing Ecológico, ya sea en publicidad, distribución o en los mismos productos, entre las cuales se destacan, Hewlett Packard, Toyota, IBM, Walmart, Coca Cola, IKEA y Phillips. Cabe destacar, que existe una práctica conocida como Greenwhashing, la cual consiste en el uso engañoso del Marketing Verde por parte de empresas, haciendo creer al consumidor que sus productos son amigables con el medio ambiente, cuando en realidad no lo son.

A nivel mundial, hoy en día estamos frente a un consumidor cada vez más informado sobre los productos ofrecidos y su impacto en el medio ambiente (Pedreros, 2014). A pesar de esto, en Chile se presenta una escasez de información e investigación respecto al marketing ecológico aplicado en bienes de consumo; surgiendo dos interrogantes claves: ¿Cuál es el grado de conciencia ecológica del consumidor? y ¿Cómo influye esto en la decisión de compra? 
Esta investigación se enfoca en la aplicación del Marketing Ecológico en bienes de consumo, tales como productos reciclados, de origen orgánico y amigables con el medio ambiente; siendo un aporte para la comunidad y empresarios que se están incorporando recién a estas nuevas prácticas de marketing.

\section{MARCO TEÓRICO}

\section{Marketing Ecológico en bienes de consumo}

La preocupación del consumidor por el medio ambiente nace después de que los medios propagaran la crisis ambiental reconocida por científicos. Con esto, los consumidores comenzaron a protestar en contra de las empresas que no actuan de manera respetuosa con el medio ambiente ni con sus derechos, obligando a los gobiernos a crear leyes que limitaran las actividades de las organizaciones contaminantes (Fraj \& Martinez, 2002). Es asi, como a mediados de los 80, las empresas incluyeron en sus prácticas de marketing el cuidado del medio ambiente, iniciando la producción y comercialización de productos ecológicos (Peattie \& Charter, 2003).

Según Chamorro (2001), el Marketing Ecológico se puede conceptualizar desde dos enfoques: un enfoque social y un enfoque empresarial. Desde un enfoque social, el Marketing Ecológico se refiere al conjunto de actividades realizadas por instituciones sin fines de lucro, con la finalidad de difundir ideas o cambiar comportamientos relacionados con el cuidado del medio ambiente. Por otra parte, el Marketing Ecológico desde un enfoque empresarial se puede definir como el conjunto de políticas que tienden a satisfacer las necesidades de los consumidores y la sociedad, de forma rentable y causando el mínimo impacto negativo sobre el medio ambiente. 
El Marketing Ecológico implica la creación de una cultura organizacional, donde no sólo se busque satisfacer las necesidades económicas de los accionistas, sino que también se busque la satisfacción de las necesidades medioambientales, sociales y económicas de los diferentes Stakeholders (accionistas, trabajadores, clientes, proveedores, competidores, comunidad, ONG's, entre otros) (Vicente, 2014).

Mayoritariamente el Marketing Ecológico es relacionado únicamente con la publicidad y promoción de productos con características ambientales (reciclables, recargables, amigables con el medio ambiente, libre de fosfato, libre de BPA, etcétera), pero este concepto va más allá de esta simple definición. Éste puede ser aplicado a la comercialización verde de bienes de consumo, bienes industriales e incluso servicios (Polonsky, 1994).

El marketing verde aplicado a la comercialización de bienes de consumo incorpora varias actividades, tales como; modificación de productos, cambios en el proceso de producción, cambios en el embalaje y modificaciones en la publicidad (Polonsky, 1994). Éste también implica la consideración del ciclo de vida del producto, de manera que su creación y diseño, la extracción de materias primas necesarias para su elaboración, el proceso productivo, distribución y consumo, o la eliminación segura de los residuos al final de su vida útil; estén orientados a generar el mínimo impacto sobre el medio ambiente (Vicente, 2014).

\section{Comportamiento del consumidor ecológico}

El comportamiento del consumidor ecológico es considerado un fenómeno complejo, en el que intervienen variables propias del individuo y variables externas a éste. Dentro de las variables internas, se encuentran valores, ideas, opiniones, personalidad y actitud que el individuo muestra ante cualquier actividad que 
desarrolla. Dentro de las variables externas, se puede encontrar la información, publicidad, amigos, familia, educación, entre otros; los cuales influirán en que la conducta del individuo sea más o menos ecológica. Un consumidor se comporta de manera ecológica cuando muestra un interés por el medio ambiente, cuando actúa de manera amigable con el medio, cuando participa en su mejora o incluso cuando está dispuesto a pagar más por un producto que es menos contaminante para el medio ambiente (Fraj \& Martinez, 2002).

El análisis del comportamiento ecológico puede centrarse en la medición del grado de interés por el medio ambiente o por los problemas medioambientales, así como también puede centrarse en el análisis de una conducta de reciclaje de ciertos materiales o de la compra de productos amigables con el medio ambiente (Fraj \& Martinez, 2002).

Según Jansson et al. (2010) indican que existen dos tipos de comportamientos del consumidor verde: restricción verde y el consumo de productos verdes. En su estudio se analiza el comportamiento frente al uso de los automóviles, una conducta restrictiva sería la de utilizar lo menos posible el automóvil y una conducta de consumo verde sería el uso de un vehículo amigable con el medio ambiente. Los resultados de este estudio indican que un bajo comportamiento ecológico no sólo es resultado de las creencias, valores y normas existentes, sino que también es resultado de la baja innovación en productos ecológicos.

Existen estudios que han indagado sobre los problemas ambientales y que han analizado el interés de los consumidores hacia productos ecológicos y hacia el cuidado del medio ambiente (Kinnear \& Taylor, 1973; Grunert \& Rohme, 1992). Además, se pueden encontrar trabajos que valoran el compromiso del consumidor 
a través de su actitud positiva hacia el reciclaje o hacia la compra de productos menos contaminantes (Calomarde, 1995; Ling-yee, 1997).

La Fundación Universidad Nacional Autónoma de México (UNAM, 2013), indicó que los países más limpios del planeta son Islandia, Suiza, Costa Rica, Suecia, Noruega, la República de Mauricio, Francia, Austria, la isla de Cuba y Colombia. Por el contrario, Estados Unidos, China, Rusia, India, Japón, Alemania, Australia, Sudáfrica, Reino Unido y Corea del Sur, son los países con mayores emisiones contaminantes. El estudio se realizó en base a indicadores como: calidad del aire, calidad del agua, emisión de gases contaminantes, políticas que aseguran la conservación del medio ambiente, protección de hábitat y utilización de energías renovables. De acuerdo al Fondo Mundial para la Naturaleza (2015), Suiza es líder mundial en consumo de productos ecológicos, con un gasto medio por persona y año de 210 euros, ratificando la tendencia actual por el consumo de estos productos.

\section{Productos que utilizan Estrategias del Marketing Ecológico}

Desde el año 2014, los productos orgánicos generaron un interés muy importante en los consumidores. Algunas de las características que exigen o buscan los consumidores en estos productos son, desde un cultivo certificado, pasando por embalajes reciclables y modelos de trabajos socialmente responsables. Hoy en día, las empresas se han adecuado a estas nuevas formas de compra, en que no basta con vender el producto, si no, que detrás debe haber una buena estrategia y un compromiso de la marca por cuidar el medio ambiente en sus procesos productivos.

La transformación y envasado de los alimentos y bebidas orgánicas es un sector muy importante del mercado orgánico. Una gran cantidad de alimentos se 
comercializan con muy poca o ninguna transformación, es decir, se realiza la trasformación primaria y su envasado, adecuándolos para su venta. La trasformación secundaria se genera en un número muy pequeño de productos y en general se realiza por el mismo supermercado o tienda especializada para su venta en el mercado interno (ProArgentina, 2005).

Chile es un país que exporta e importa una gran cantidad de productos, dentro de esta gama, existen productos orgánicos. Se exportan alrededor de 70 tipos de productos orgánicos y son importados más de 30 tipos (Oficina de Estudios y Políticas Agrarias, 2014). Las importaciones más importantes en Chile en 2014 fueron los ajos frescos o refrigerados, té verde, café y aceite de oliva virgen. Los países de donde provienen estos productos son China, Argentina, Estados Unidos y España. Dentro de productos que más se exportaron en 2014 fueron los arándanos frescos, seguido por las manzanas frescas y los arándanos congelados. El principal país de destino para estos productos fue Estados Unidos, país al que se enviaron más de 27 mil toneladas de productos.

Según Reinhardt (1998), la diferenciación de productos amigables con el medio ambiente tiene lugar cuando una empresa crea productos que proporcionan mayores beneficios ambientales, o que imponen menores costes ambientales, que los productos similares. Esta definición señala, que los productos ecológicos no son sólo aquellos productos con un menor impacto ambiental, sino también aquellos que proporcionan beneficios ambientales más altos en comparación con los productos convencionales.

El patrón de consumo de los consumidores ha cambiado debido al crecimiento económico de la degradación del medio ambiente (Fraj \& Martinez, 2002). Esta preocupación ha inducido el desarrollo de los vendedores verdes hacia las 
estrategias de marketing ecológico que causarían menos daño al medio ambiente (Maheswari, 2015). Las empresas dan énfasis a los procesos de producción eficientes (en términos de reducción de agua, uso de materias primas y producción de residuos). También se hacen esfuerzos importantes para reducir el tamaño y peso de los productos, componentes y embalaje. Con el fin de tener un impacto "nulo" algunas empresas desarrollan varios productos (tales como ropa, calzado, materiales de construcción) sólo utilizando materiales reciclados o naturales (como bioplástico y algodón orgánico) (Polonsky, 1994; Dangierico \& Pontrandolfo, 2010).

Seleccionar productos con bajo impacto ambiental aporta una serie de ventajas, tanto medioambientales como sociales y/o económicas (Bastante et al., 2001). En cuanto a las ventajas medioambientales, cabe destacar las siguientes:

- Ahorro de recursos naturales, tanto renovables como no renovables.

- Ahorro de energía.

- Disminución en el volumen y naturaleza de compuestos tóxicos empleados en productos y procesos.

Respecto a las ventajas económicas y/o sociales:

- Disminución del riesgo sobre la salud de la población debido a un menor uso de sustancias peligrosas y/o contaminantes.

- Promoción de cambios culturales.

- Mayor calidad de vida de los ciudadanos.

- Mayor influencia del mercado para promocionar nuevas marcas, soluciones técnicas, empresas y puestos de trabajo. 
- Transformación de residuos en subproductos.

\section{Validación de normas ecológicas}

En el último tiempo, de acuerdo a la creciente conciencia global por proteger al medio ambiente y la necesidad de orientación en una compra sustentable, nace el concepto de Eco-etiquetas o Etiquetas Verdes (D'Souza, 2004). Éstas se definen como símbolos que se otorgan a aquellos productos que tienen un impacto menor sobre el medio ambiente, de acuerdo al cumplimiento de una serie de criterios ecológicos, ya sea basado en su ciclo de vida o en algunas características más puntuales (CEGESTI, 2013). Para lo cual, existen dos categorías de clasificación: voluntarias y obligatorias (Gazulla, 2010).

Para el caso de las etiquetas voluntarias, se pueden encontrar tres tipos de ecoetiquetas presentes en el mercado. Las de tipo I informan al consumidor de manera fácil y sencilla por medio de un logotipo, sobre las ventajas ambientales del bien de consumo; además, son certificadas por una tercera parte independiente, habitualmente una administración pública ambiental (Senerman \& Aguirre, 2015). En la Imagen 1 se presentan algunos ejemplos.

\section{Imagen 1: Etiquetas tipo I}




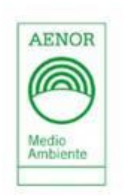

AENOR medioambiente

(España)

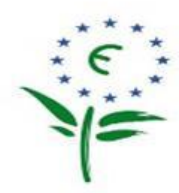

Etiqueta ecológica Europea (Ecolabel)

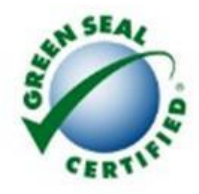

Green Seal

(USA)

Fuente: Elaboración propia a partir de Anales de la Universidad Metropolitana (2011)

Las etiquetas tipo II, también denominadas auto-declaraciones medioambientales, pueden ser efectuadas por los propios fabricantes, importadores, distribuidores, detallistas y cualquier otro actor que se beneficie de dicha declaración (Penela, 2010), sin ser verificadas por organismos independientes (Bastante et al., 2011). En la Imagen 2 se presentan algunos ejemplos.

\section{Imagen 2: Etiquetas tipo II}

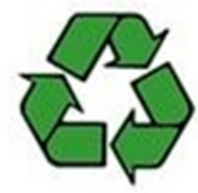

Triangulo de Moebius (reciclado)

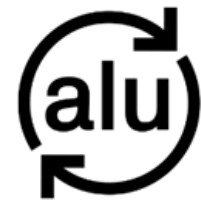

Aluminio reciclable

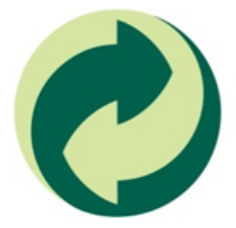

Gestión de envases

Fuente: Elaboración propia a partir de Anales de la Universidad Metropolitana (2011)

Finalmente, las etiquetas de tipo III entregan información de los impactos ambientales que un producto tiene durante todo su ciclo de vida, siendo aquella etiqueta que brinda más información sobre el impacto ambiental de un proceso o servicio, y a la vez la más compleja de entender, convirtiéndose en una desventaja para aquellos consumidores que no están preparados para utilizar información 
técnica en su decisión de compra (CEGESTI, 2013). A continuación, se presenta un ejemplo de eco-etiqueta tipo III.

Imagen 3: Etiquetas tipo III

\begin{tabular}{|c|c|}
\hline & $\begin{array}{r}\text { Resumen } \\
\begin{array}{r}\text { Declaración Ambiental } \\
\text { de Producto }\end{array}\end{array}$ \\
\hline $\begin{array}{l}\text { Institut Bauen und Umwelt e.V. } \\
\text { www.bau-umwelt.com }\end{array}$ & Intervención en el programa \\
\hline $\begin{array}{l}\text { EGGER Retail Products GmbH \& Co. KG } \\
\text { Im Kissen } 19 \\
\text { D-59929 Brilon }\end{array}$ & Titular de la Declaración \\
\hline EPD-EHW-2008211-ES & Número de Declaración \\
\hline $\begin{array}{l}\text { Suelo laminado de Egger Retails Products, clases de uso } 31,32 \text { y } 33 \text { (AC3 - AC5) } \\
\text { La presente declaración constituye una Declaración Ambiental de Producto conforme a la norma ISO } \\
14025 \text { y describe el comportamiento ambiental de los productos de construccín aqui mencionados. } \\
\text { Su objetivo consiste en fomentar el desarrollo de una construcción compatible con la protección } \\
\text { ambiental y sanitaria. } \\
\text { En esta declaración certificada se exponen todos los datos ambientales pertinentes. La declaración } \\
\text { se basa en el documento de PCR (Reglas de categoria de producto) 'Holzwerkstoffe' relativo a } \\
\text { materiales derivados de la madera, ano de referencia } 2007 \text {. }\end{array}$ & $\begin{array}{r}\text { Productos de construcción } \\
\text { declarados }\end{array}$ \\
\hline $\begin{array}{l}\text { Esta declaración certificada autoriza a su titular a utilizar el simbolo del "Institut Bauen und Umwelt: } \\
\text { Es valida únicamente para los productos mencionados y por un periodo de un año a partir de la } \\
\text { fecha de expedición. } \\
\text { El titular de la declaración es responsable de la información y la documentación que sirven de base. }\end{array}$ & Validez \\
\hline $\begin{array}{l}\text { La declaración está completa y contiene de forma detallada: } \\
\text { - Definición del producto y caracteristicas fisicas } \\
\text { - Información sobre las materias primas y sobre su origen } \\
\text { - Especificaciones sobre la fabricación del producto } \\
\text { - Referencias sobre el procesamiento del producto } \\
\text { - Información sobre estado de uso, efectos extraordinarios y fase de fin de vida } \\
\text { - Resultados del análisis del ciclo de vida } \\
\text { - Documentación y pruebas }\end{array}$ & Contenido de la declaración \\
\hline
\end{tabular}

Fuente: EGGER Retail Products GmbH \& Co. KG (2011)

Por otro lado, las eco-etiquetas obligatorias son aquellas cuyo uso está establecido por ley, informando del comportamiento ambiental de un producto durante una fase del ciclo de vida y permitiendo realizar al consumidor final, una comparación sencilla entre diferentes modelos del mercado en base a sus características amigables con el medio ambiente (Consejería de Medio Ambiente y Ordenación de Territorio, 
2011). Un claro ejemplo de este tipo de eco-etiquetas es la conocida etiqueta Europea utilizada en productos domésticos, vehículos o edificios; la cual obliga a dar a conocer el comportamiento ambiental del producto en base a una letra que mide la eficiencia energética (Imagen 4).

\section{Imagen 4: Etiquetas Europeas}

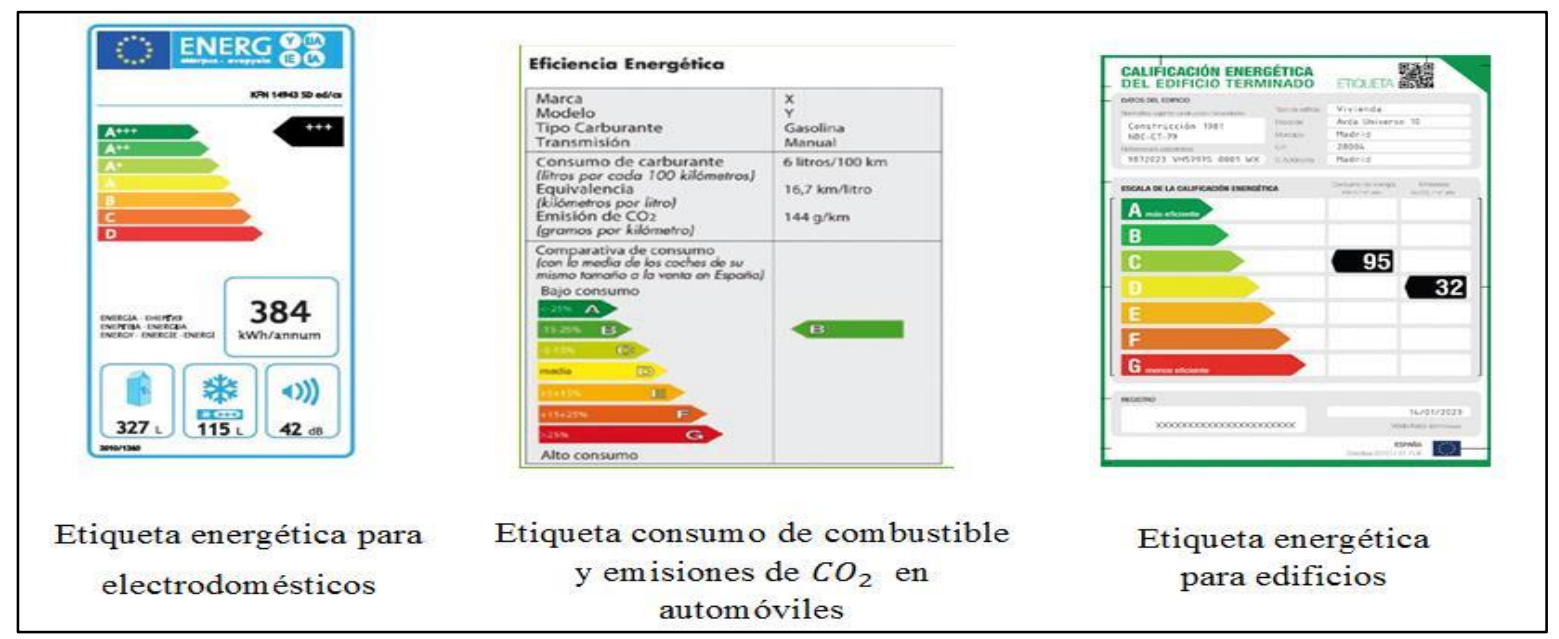

Fuente: Elaboración propia a partir de Consejería de Medio Ambiente y Ordenación de

\section{Territorio (2011)}

En el caso de Chile, a diferencia de otros países, aún no existe una eco-etiqueta oficial, es decir, eco-etiqueta diseñada, certificada y estandarizada en el país. Por ende, no existe un organismo oficial que regule estas categorías (Servicio Nacional del Consumidor, 2012). En cambio, según un estudio presentado por la Fundación Chile (2014), existen en el país alrededor de 21 eco-etiquetas no oficiales para 
distintos grupos de productos, en los cuales se destacan los productos alimenticios, productos forestales y materiales de construcción.

\section{Greenwashing}

El principal objetivo del eco-etiquetado es asegurar una información ambiental real y fiable, evitando el Greenwashing, es decir, evitando engaños en la entrega de información ambiental en productos.

Las empresas están cada vez más enfocadas en proyectar una imagen amigable con el medio ambiente frente a los consumidores, como consecuencia del alto grado de preocupación ambiental que poseen estos hoy en día, pero esta imagen no siempre refleja la realidad. Ante esta situación, aparece el concepto de Greenwashing, término usado para describir la práctica de ciertas compañías que le dan un giro a la presentación de sus productos y/o servicios para hacerlos ver como respetuosos del medio ambiente. No obstante, este giro es meramente de forma y no de fondo, por lo que se convierte en un uso engañoso de la comercialización verde (Comunicación de Sustentabilidad y RSE, 2009).

En Chile, el único organismo gubernamental que se encarga del monitoreo de la publicidad de las empresas con respecto a esta práctica llamada Greenwashing, es el Servicio Nacional del Consumidor a través de la Ley №19.496, especialmente en lo que respecta la comprobabilidad y veracidad de la información, tanto en mensajes publicitarios, como en sellos o en certificaciones que son presentados al público consumidor (Servicio Nacional del Consumidor, 2012).

\section{METODOLOGÍA}

\section{Diseño y procedimiento}


Se aplicó una encuesta con el propósito de identificar el grado de conciencia ecológica en los individuos y a la vez, ver si esta conciencia incidía en la decisión de compra de bienes de consumo.

Con el fin de que los individuos tuvieran presente una compra actual realizada, la encuesta se realizó de forma presencial y directa a los consumidores en las inmediaciones de los distintos supermercados y tiendas de retail tales como Lider, Homecenter Sodimac, Easy, Jumbo, Santa Isabel, Unimarc, Falabella y Almacenes Paris.

La encuesta estuvo compuesta por un total de trece preguntas, donde se midieron principalmente cuatro variables, siendo éstas la conciencia ecológica del consumidor, la intención de compra frente a productos ecológicos, su comportamiento hacia los productos ecológicos, y su conocimiento y credibilidad hacia estos productos. Para la medición de las variables se analizaron diferentes dimensiones por medio de escalas tipo Likert ( 1 a 5 puntos). Al final del cuestionario se midieron ciertas variables sociodemográficas. En la Tabla 1 se indican las variables usadas y su correspondiente fuente.

Tabla 1. Variables del cuestionario, tipo de pregunta y fuente 


\begin{tabular}{|l|l|l|}
\hline \multicolumn{1}{|c|}{ Variable } & \multicolumn{1}{|c|}{ Tipo de pregunta } & \multicolumn{1}{|c|}{ Fuente } \\
\hline P.1 Conciencia ecológica & Likert 5 puntos & Bohlen et al. (1993) \\
\hline P.2 Intención de compra & Likert 5 puntos & $\begin{array}{l}\text { Dunlap y Van Liere (1978) } \\
\text { Webb et al. (2007) }\end{array}$ \\
\hline $\begin{array}{l}\text { P.3 Comportamiento hacia los } \\
\text { productos ecológicos }\end{array}$ & Likert 5 puntos & $\begin{array}{l}\text { Maloney y Ward (1985) } \\
\text { Karp (1996) }\end{array}$ \\
\hline $\begin{array}{l}\text { P.4 a P.5 Productos y marcas } \\
\text { ecológicas recordada por el } \\
\text { consumidor }\end{array}$ & Preguntas abiertas & \\
\hline $\begin{array}{l}\text { P.6 Conocimiento y } \\
\text { credibilidad hacia los productos } \\
\text { ecológicos } \\
\text { P.7 Variables que inciden en la } \\
\text { decisión de compra }\end{array}$ & Likert 5 puntos & $\begin{array}{l}\text { Weigel y Weigel (1978) } \\
\text { Easterling, Miller y Weinberger (1995) }\end{array}$ \\
\hline $\begin{array}{l}\text { P.8 a P.13 Variables } \\
\text { sociodemográficas }\end{array}$ & - & Grunert y Kristensen (1992) \\
\hline
\end{tabular}

Fuente: Elaboración propia

\section{Descripción de la muestra}

La encuesta fue aplicada a un total de 351 personas con edades entre los 18 a 64 años, pertenecientes a Chile, ya que a partir de los 18 años se comienza a percibir ingresos propios, teniendo poder en su decisión de compra y finalizando a los 64 años, debido a que a esta edad la mayoría de las personas se jubilan, disminuyendo su poder adquisitivo, razón por la que dejan de considerar marcas al momento de la compra. La edad promedio de la muestra fue de 31 años, el $54 \%$ de los participantes fueron hombres y el $68 \%$ de los individuos tenian estudios superiores (Tabla 2). 
Tabla 2. Característica de la muestra

\begin{tabular}{lcc}
\hline Variable & Frecuencia & Porcentaje \\
GENERO & 161 & \\
Mujer & 190 & $46 \%$ \\
Hombre & & $54 \%$ \\
RANGO DE INGRESOS & 151 & \\
Menor a $\$ 250.000$ & 116 & $43 \%$ \\
Entre $\$ 250.00$ a $\$ 500.000$ & 81 & $33 \%$ \\
Mayor a $\$ 500.000$ & & $23 \%$ \\
NIVEL DE ESTUDIO & 239 & $68 \%$ \\
Con estudio superior & 112 & $32 \%$ \\
Sin estudio superior & & \\
EDAD & 190 & $54 \%$ \\
18 a 27 años & 81 & $23 \%$ \\
28 a 40 años & 81 & $23 \%$ \\
41 a 64 años & 351 & $\mathbf{1 0 0}$ \\
\hline TOTAL & & \\
\hline
\end{tabular}

\section{RESULTADOS}

Existen diversos coeficientes para medir la fiabilidad de una escala, siendo el coeficiente alfa de Cronbach el más utilizado en las ciencias sociales (Zumbo \& Rupp, 2004), ya que es la forma más sencilla de medir la consistencia interna de una escala y su primera aproximación a la validación. Las recomendaciones para evaluar los coeficientes del alfa de Cronbach tienen escalas de aceptación a partir de 0,5. Autores como George y Mallery (1994) sugieren que un Alfa de 0,5 es aceptable aunque su valor sea bajo, mientras que Numally y Bernstein (1994) 
afirman que el valor de fiabilidad en investigación exploratoria debe ser igual o mayor a 0,7 .

Tras analizar las diferentes escalas a través del coeficiente de alfa de Cronbach se obtuvo que la mayoría de éstas se encontraban en un rango superior a 0,60 a excepción de la escala de comportamiento ecológico, la cual presentó un coeficiente de 0,515 , valor aceptable según los autores George y Mallery (1994). Con respecto a los ítems de cada variable, quienes obtuvieron una mayor media fueron; para el caso de conciencia ecológica "Hago el mejor esfuerzo para proteger el medio ambiente" con una media de 3,89; en intención de compra las personas asignaron una mayor media a la afirmación "Si tuviera la oportunidad, compraría productos que estuviesen hechos con materiales reciclado o que pudiesen reutilizarse" con 4,53; en comportamiento ecológico se pudo observar que las personas realizan con mayor frecuencia la compra de ampolletas de ahorro energético, teniendo una media de 4,46; mientras que para variable conocimiento y credibilidad hacia productos ecológicos los consumidores declararon conocer los lugares pueden encontrar productos ecológicos, al presentar una media de 3,49. Estos resultados se pueden ver en la Tabla 3.

Tabla 3. Fiabilidad de las variables de interés

\begin{tabular}{lcc}
\hline Variable & $\begin{array}{c}\text { Alfa De } \\
\text { Cronbach }\end{array}$ & Media \\
\hline CONCIENCIA ECOLÓGICA & 0,717 & 3,82 \\
Hago el mejor esfuerzo para proteger el medio ambiente & & 3,89 \\
\hline $\begin{array}{l}\text { INTENCIÓN DE COMPRA } \\
\begin{array}{l}\text { Si tuviera la oportunidad, compraría productos que } \\
\text { estuviesen hechos con materiales reciclados o que } \\
\text { pudiesen reutilizarse }\end{array}\end{array}$ & 0,778 & 4,18 \\
& & 4,53 \\
& 0,611 & 3,05
\end{tabular}

REDMARKA UIMA-Universidad de A Coruña - CIECID

Año X, Número 18, (2017), v I pp. 49-82

http://www.redmarka.net/ ISSN 1852-2300 
¿CÓMO AFECTA LA CONCIENCIA ECOLÓGICA DEL INDIVIDUO AL MOMENTO DE LA COMPRA DE BIENES DE CONSUMO? UN ESTUDIO APLICADO A CHILE

Hidalgo-Alcázar, Carmen

\begin{tabular}{|c|c|c|}
\hline $\begin{array}{l}\text { CONOCIMIENTO Y CREDIBILIDAD HACIA LOS } \\
\text { PRODUCTOS ECOLÓGICOS }\end{array}$ & & \\
\hline $\begin{array}{l}\text { Conozco los lugares donde puedo encontrar productos } \\
\text { ecológicos }\end{array}$ & & 3,49 \\
\hline COMPORTAMIENTO ECOLÓGICO & 0,515 & 3,41 \\
\hline Compro ampolletas de ahorro energético & & 4,46 \\
\hline
\end{tabular}

\section{Relación entre las variables cociencia ecologica, intención de compra y comportamiento ecologico.}

Para dar mayor riqueza a la investigación, se aplicó el método de Preacher y Hayes (2008) que determina la relación existente entre las variables conciencia ecológica, intención de compra y comportamiento ecológico. Los resultados mostrarón una relación positiva y significativa entre la conciencia ecológica y la intención de compra $(\beta=0,48 ; p<0,01)$; y entre la intención de compra y el comportamiento ecológico $(\beta=0,23 ; p<0,01)$. Además, el efecto directo entre conciencia y comportamiento ecológico, resulto ser significativo $(\beta=0,29 ; p<0,01)$. Como se muestra en la Figura 1 , el efecto indirecto que tiene la conciencia sobre el comportamiento ecológico, a través de la intención de compra, es significativo con un intervalo de confianza del $95 \%$ excluyendo el cero $(0,0610$ a 0,1739$)$. Estos resultados sugieren que el efecto de la conciencia ecológica en el comportamiento ecológico está parcialmente mediado a través de la intención de compra.

Figura 1. Efectos directos e indirectos 


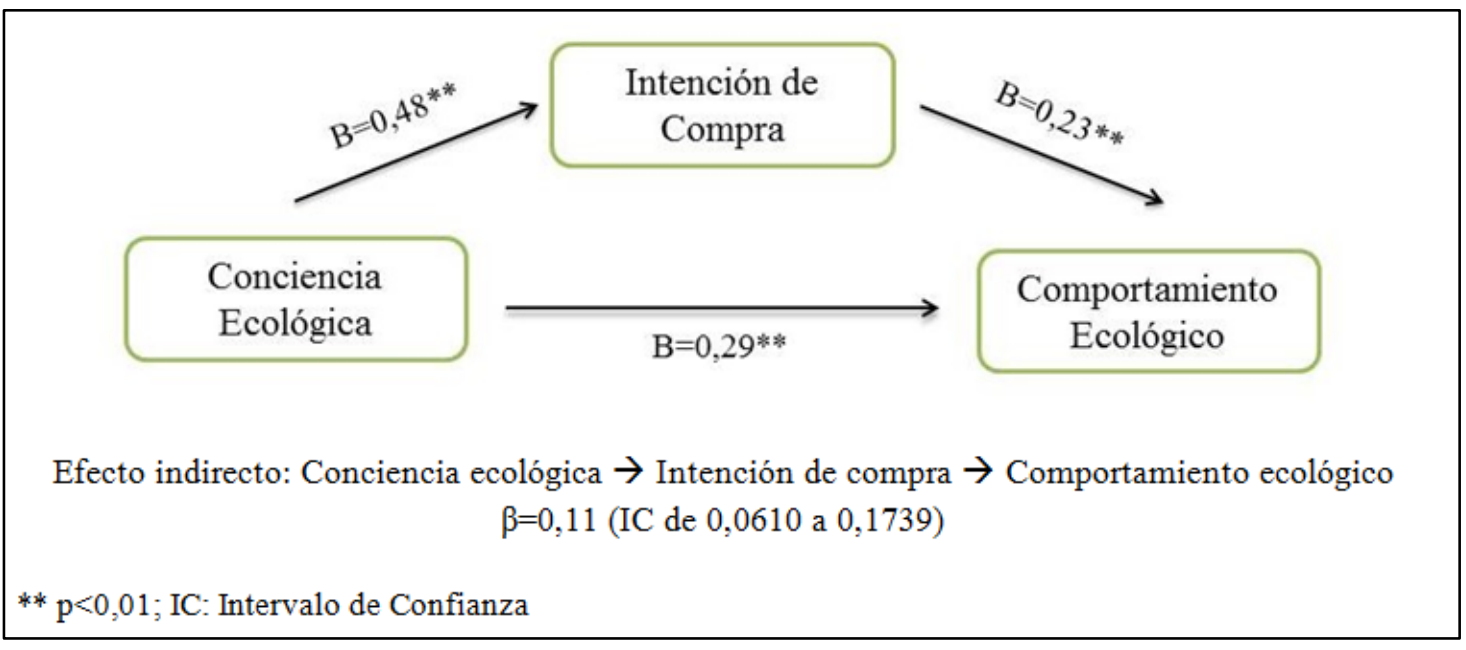

Fuente: Elaboración propia

Además, se realizó un Analisis de la Varianza (ANOVA) para determinar el efecto que tienen las características sociodemográficas sobre las variables conciencia ecológica, intención de compra y comportamiento ecológico.

Se observaron diferencias estadísticamente significativas en el nivel de conciencia ecológica en función de la edad, destacando que las personas con edad entre 41 y 64 años son las que presentan mayor conciencia. Los contrastes post hoc revelan que las diferencias son significativas entre todos los grupos de edad (Tabla 4). Además, las variables rango de ingresos y nivel de estudios también influyen en el nivel de conciencia ecológica. Las diferencias significativas respecto al rango de ingresos se observan entre los de ingresos menores a $\$ 250.000$ y mayor a $\$ 500.000$. Es decir, a medida que se posee un mayor poder adquisitivo, se tiene una mayor conciencia ecológica. Por otro lado, a mayor nivel de estudios la conciencia ecológica disminuye (Tabla 4).

\section{Tabla 4 Conciencia Ecológica}


¿CÓMO AFECTA LA CONCIENCIA ECOLÓGICA DEL INDIVIDUO AL MOMENTO DE LA

COMPRA DE BIENES DE CONSUMO? UN ESTUDIO APLICADO A CHILE

Hidalgo-Alcázar, Carmen

\begin{tabular}{|c|c|c|c|c|c|}
\hline Variable independiente & \multicolumn{4}{|c|}{$\begin{array}{c}\text { CONCIENCIA ECOLÓGICA } \\
\text { Desv. }\end{array}$} & $\begin{array}{l}\text { Post- } \\
\text { hoc }\end{array}$ \\
\hline \multicolumn{6}{|l|}{ EDAD } \\
\hline (1) 18-27 años & 3,57 & 0,74 & & & $(1)-(2)^{\star \star}$ \\
\hline (2) 28-40 años & 3,93 & 0,69 & 31,181 & 0,000 & $(1)-(3)^{\star \star}$ \\
\hline (3) 41-64 años & 4,32 & 0,70 & & & $(2)-(3)^{*}$ \\
\hline \multicolumn{6}{|l|}{ RANGO DE INGRESOS } \\
\hline $\begin{array}{l}\text { (1) Menor a } \$ 250.000 \\
\text { (2) Entre } \$ 250.000\end{array}$ & 3,58 & 0,80 & 14,179 & 0,000 & $(1)-(2)^{\star \star}$ \\
\hline a $\$ 500.000$ & 3,95 & 0,77 & & & $(1)-(3)^{\star \star}$ \\
\hline (3) Sobre $\$ 500.000$ & 4,07 & 0,64 & & & \\
\hline \multicolumn{6}{|l|}{ NIVEL DE ESTUDIOS } \\
\hline (1) Sin estudios superiores & 4,04 & 0,82 & 14,152 & 0,000 & $\mathrm{np}$ \\
\hline (2)Con estudios superiores & 3,71 & 0,74 & & & \\
\hline
\end{tabular}

En la Tabla 5 se pueden ver las diferencias significativas de la intención de compra respecto del género, edad y rango de ingresos. Se observó que las mujeres tienen una mayor intención de compra que los hombres, con una media de 4,32 y 4,07, respectivamente. Por otro lado, a medida que aumenta la edad aumenta la intención de compra, siendo las diferencias significativas entre jóvenes y adultos, y entre los de mediana y mayor edad.

En cuanto a la variable rango de ingreso, es curioso que se destacan con mayor intención de compra aquellas personas que poseen un ingreso entre $\$ 250.000$ y $\$ 500.000$. Las diferencias significativas se encuentran entre los de bajo y mediano ingreso, y entre los de bajo y alto ingreso, a un nivel de significatividad del $1 \%$ y $5 \%$ respectivamente (Tabla 5 ).

Tabla 5. Intención de compra 


\begin{tabular}{|c|c|c|c|c|c|}
\hline & Medias & $\begin{array}{l}\text { Desv. } \\
\text { Típica }\end{array}$ & $\mathbf{F}$ & Sig. & Post-hoc \\
\hline \multicolumn{6}{|l|}{ GÉNERO } \\
\hline (1) Hombre & 4,07 & 0,77 & 9,755 & 0,002 & $\mathrm{~Np}$ \\
\hline (2) Mujer & 4,32 & 0,68 & & & \\
\hline \multicolumn{6}{|l|}{ EDAD } \\
\hline (1) $18-27$ años & 4,02 & 0,77 & 13,854 & 0,000 & $(1)-(3)^{\star *}$ \\
\hline (2) 28-40 años & 4,23 & 0,73 & & & $(2)-(3)^{*}$ \\
\hline (3) 41-64 años & 4,52 & 0,54 & & & \\
\hline \multicolumn{6}{|l|}{ RANGO DE INGRESOS } \\
\hline \multirow{2}{*}{$\begin{array}{l}\text { (1) Menor a } \$ 250.000 \\
\text { (2) Entre } \$ 250.000 \\
\text { a } \$ 500.000\end{array}$} & 4,03 & 0,77 & 6,095 & 0,000 & $(1)-(2)^{* *}$ \\
\hline & 4,31 & 0,71 & & & $(1)-(3)^{*}$ \\
\hline (3) Sobre $\$ 500.000$ & 4,29 & 0,67 & & & \\
\hline
\end{tabular}

En la Tabla 6, se observan las diferencias de la variable comportamiento hacia los productos ecológicos y las variables género, edad, rango de ingresos y nivel de estudios. Los resultados muestran que presentan un mayor comportamiento ecológico las mujeres $(3,51)$, las personas que tienen entre 41-64 años de edad $(3,71)$, aquellos con ingresos sobre los $\$ 500.000(3,57)$ y las personas sin estudios superiores $(3,66)$. Dentro de la variable edad, los contrastes post hoc revelan que las diferencias son significativas entre los de menor y mediana edad, y entre los de menor y mayor edad. Con respecto al rango de ingresos, las diferencias se encuentran en los de bajo y mediano ingreso, y entre los de bajo y alto ingreso a una significancia del $5 \%$ y $1 \%$ respectivamente.

Tabla 6. Comportamiento ecológico

\begin{tabular}{llllll}
\hline Variable independiente & \multicolumn{4}{c}{ COMPORTAMIENTO ECOLÓGICO } \\
& Medias & Desv. Típica & F & Sig. & Post- \\
\hline
\end{tabular}




\section{GÉNERO}
(1) Hombre
3,34
0,72
4,825
$0,029 \mathrm{~Np}$
(2) Mujer
3,51
0,73

EDAD
(1) 18-27 años
$3,24 \quad 0,69$
14,826
0,000
$(1)-(2)^{\star *}$
(2) 28-40 años
3,56
0,71
$(1)-(3)^{\star *}$
(3) 41-64 años
3,71
0,70

\section{RANGO DE INGRESOS}

$\begin{array}{llll}\text { (1) Menor a } \$ 250.000 & & 3,27 & 0,68 \\ \text { (2) Entre } \$ 250.000 & \text { a } & & \\ \$ 500.000 & & 3,5 & 0,72 \\ \text { (3) Sobre } \$ 500.000 & 3,57 & 0,77\end{array}$

NIVEL DE ESTUDIOS

(1) Sin estudios superiores $3,66 \quad 0,71$

(2)Con estudios superiores $3,31 \quad 0,71$

${ }^{*} \mathrm{p}<0,05 ;{ }^{* *} \mathrm{p}<0,01 ; \mathrm{np}$ : no procede

En la Tabla 7 se observa que existen diferencias significativas en el conocimiento y credibilidad hacia los productos ecológicos en función del género, la edad y el nivel de estudios, con un nivel de significatividad del $5 \%$. Se puede destacar que quienes tienen mayor conocimiento y credibilidad hacia los productos ecológicos son las mujeres $(3,19)$, los adultos jóvenes con edades entre 28 y 40 años $(3,07)$ y las personas con estudios superiores $(3,13)$. Es importante mencionar que las diferencias significativas en la edad se encuentran únicamente entre los de menor y mayor edad, es decir, entre personas con rango de edad de 18-27 años y 41-64 años. 
¿CÓMO AFECTA LA CONCIENCIA ECOLÓGICA DEL INDIVIDUO AL MOMENTO DE LA

COMPRA DE BIENES DE CONSUMO? UN ESTUDIO APLICADO A CHILE

Hidalgo-Alcázar, Carmen

Tabla 7. Conocimiento y credibilidad como variable dependiente

\begin{tabular}{|c|c|c|c|c|c|}
\hline \multirow[t]{2}{*}{ Variable independiente } & \multicolumn{5}{|c|}{ CONOCIMIENTO Y CREDIBILIDAD } \\
\hline & Medias & Desv. Típica & $\mathbf{F}$ & Sig. & Post-hoc \\
\hline \multicolumn{6}{|l|}{ GÉNERO } \\
\hline (1) Hombre & 2,94 & 0,85 & 7,123 & 0,008 & $\mathrm{~Np}$ \\
\hline (2) Mujer & 3,19 & 0,91 & & & \\
\hline \multicolumn{6}{|l|}{ EDAD } \\
\hline (1) 18-27 años & 3,14 & 0,78 & 3,188 & 0,042 & $(1)-(3)^{*}$ \\
\hline (2) 28-40 años & 3,07 & 0,94 & & & \\
\hline (3) 41-64 años & 2,84 & 1,04 & & & \\
\hline \multicolumn{6}{|l|}{ NIVEL DE ESTUDIOS } \\
\hline (1) Sin estudios superiores & 2,90 & 1,01 & 5,282 & 0,022 & $\mathrm{~Np}$ \\
\hline (2)Con estudios superiores & 3,13 & 0,81 & & & \\
\hline
\end{tabular}

\section{Análisis de variables que influyen en la decisión de compra}

A la hora de comprar productos, existen diferentes variables que influyen en la selección de éste; tales como la calidad, el precio, la etiqueta, el empaque o el diseño y la marca. Según los resultados de los descriptivos para esta variable, el consumidor le da mayor importancia a la calidad $(4,68)$, seguido del precio $(3,99)$ y de la etiqueta $(3,70)$. Destacar que la marca es la variable menos influyente en la decisión de compra (2.56) (Tabla 8).

Tabla 8. Variables que influyen en la decisión de compra

\begin{tabular}{lr}
\hline Variable & Media \\
\hline Calidad & 4,68 \\
Precio & 3,99 \\
Etiqueta & 3,7 \\
Empaque o Diseño & 2,73 \\
Marca & 2,56 \\
\hline
\end{tabular}


A continuación, se realizaron los análisis de diferencias de las variables que influyen en la decisión de compra en función de ciertas variables demográficas.

Como se observa en la Tabla 9, quienes le dan mayor importancia a la calidad al momento de comprar un producto ecológico son personas con edades entre 41 y 64 años, evidenciando, según los contrastes post hoc, que la diferencia significativa se encuentra entre los de menor y mayor edad.

Tabla 9. Calidad

\begin{tabular}{llllll}
\hline $\begin{array}{l}\text { Variable } \\
\text { independiente }\end{array}$ & \multicolumn{5}{c}{ CALIDAD } \\
& Medias & Desv. Típica & F & Sig. & Post-hoc \\
\hline EDAD & & & & & \\
(1) $18-27$ años & 4,61 & 0,731 & 5,503 & 0,003 & $(1)-(3)^{\star *}$ \\
(2) $28-40$ años & 4,66 & 0,615 & & & \\
(3) $41-64$ años & 4,89 & 0,36 & & & \\
\hline${ }^{*} \mathrm{p}<0,05 ;{ }^{* *} \mathrm{p}<0,01$ & & & &
\end{tabular}

Según la Tabla 10, las personas que tienen un ingreso menor a $\$ 250.000$ le dan mayor importancia al precio al momento de comprar un producto ecológico, dándose la diferencia significativa entre los de bajo y alto ingreso.

Tabla 10. Precio

\begin{tabular}{|c|c|c|c|c|c|}
\hline \multirow[t]{2}{*}{ Variable independiente } & \multicolumn{5}{|c|}{ PRECIO } \\
\hline & Medias & Desv. Típica & $\mathbf{F}$ & Sig. & Post-hoc \\
\hline \multicolumn{6}{|l|}{ RANGO DE INGRESOS } \\
\hline $\begin{array}{l}\text { (1) Menor a } \$ 250.000 \\
\text { (2) Entre } \$ 250.000\end{array}$ & 4,21 & 0,95 & 6,927 & 0,001 & $(1)-(3)^{\star *}$ \\
\hline a $\$ 500.000$ & 3,99 & 1,35 & & & \\
\hline (3) Sobre $\$ 500.000$ & 3,60 & 1,39 & & & \\
\hline
\end{tabular}

REDMARKA UIMA-Universidad de A Coruña - CIECID

Año X, Número 18, (2017), v I pp. 49-82

http://www.redmarka.net/ ISSN 1852-2300 
En la Tabla 11, se puede observar el análisis de diferencias de la variable etiqueta en función de la edad, nivel de estudios y rango de ingresos. A un nivel de significatividad del $5 \%$, se observó que los adultos con edad entre 41 y 64 años $(4,28)$ son los que más se fijan en la etiqueta al momento de comprar un producto ecológico, encontrándose las diferencias entre los de menor y mayor edad, y entre los de mediana y mayor edad. Además, las personas sin estudios superiores $(4,33)$ y aquellos que tienen ingresos entre $\$ 250.000$ y $\$ 500.000(4,07)$ son también los que más se preocupan por la etiqueta al momento de comprar.

Tabla 11. Etiqueta

\begin{tabular}{|c|c|c|c|c|c|}
\hline \multirow[t]{2}{*}{ Variable independiente } & \multicolumn{5}{|c|}{ ETIQUETA } \\
\hline & Medias & Desv. Típica & $\mathbf{F}$ & Sig. & Post-hoc \\
\hline \multicolumn{6}{|l|}{ EDAD } \\
\hline (1) 18-27 años & 3,36 & 1,35 & 7,46 & 0,001 & $(1)-(3)^{\star *}$ \\
\hline (2) $28-40$ años & 3,96 & 1,44 & & & $(2)-(3)^{\star *}$ \\
\hline (3) 41-64 años & 4,28 & 1,64 & & & \\
\hline \multicolumn{6}{|l|}{ NIVEL DE ESTUDIOS } \\
\hline (1) Sin estudios superiores & 4,33 & 1,15 & 36,125 & 0,000 & $\mathrm{np}$ \\
\hline (2) Con estudios superiores & 3,41 & 1,42 & & & \\
\hline \multicolumn{6}{|l|}{ RANGO DE INGRESOS } \\
\hline (1) Menor a $\$ 250.000$ & 3,43 & 1,42 & 6,906 & 0,001 & $(1)-(2)^{\star *}$ \\
\hline (2) Entre $\$ 250.000$ a $\$ 500.000$ & 4,07 & 1,33 & & & \\
\hline (3) Sobre $\$ 500.000$ & 3,91 & 1,39 & & & \\
\hline
\end{tabular}

* $p<0,05 ;{ }^{*} p<0,01 ; n p:$ no procede

Las personas que le dan mayor importancia al empaque o diseño a la hora de comprar un producto ecológico son aquellas que tienen una edad entre 41 y 64 años $(3,23)$, existiendo la diferencia significativa entre los de menor y mayor edad, y entre los de mediana y mayor edad. Además, aquellos que no tienen estudios superiores se preocupan un poco más del empaque o diseño de los que tienen estudios superiores (Tabla 12).

Tabla 12. Empaque o Diseño 
¿CÓMO AFECTA LA CONCIENCIA ECOLÓGICA DEL INDIVIDUO AL MOMENTO DE LA

COMPRA DE BIENES DE CONSUMO? UN ESTUDIO APLICADO A CHILE

Hidalgo-Alcázar, Carmen

\begin{tabular}{llllll}
\hline Variable independiente & \multicolumn{5}{c}{ EMPAQUE O DISEÑO } \\
& Medias & Desv. Típica & F & Sig. & Post-hoc \\
\hline EDAD & & & & & \\
$\quad$ (1) $18-27$ años & 2,68 & 0,73 & 31,181 & 0,000 & $(1)-(3)^{* *}$ \\
(2) $28-40$ años & 2,36 & 0,62 & & & $(2)-(3)^{* *}$ \\
$\quad$ (3) $41-64$ años & 3,23 & 0,36 & & & \\
NIVEL DE ESTUDIOS & & & & & \\
$\quad$ (1) Sin estudios superiores & 2,98 & 1,65 & 5,011 & 0,026 & $\mathrm{np}$ \\
(2) Con estudios superiores & 2,61 & 1,36 & & & \\
\hline * p<0,05; ${ }^{* *}$ p<0,01; np: no procede
\end{tabular}

\section{CONCLUSIONES}

La presente investigación tuvo por objetivo identificar el grado de conciencia ecológica y ver si ésta influye en la decisión de compra de los consumidores, con el fin de proponer estrategias aplicables al marketing ecológico en bienes de consumo.

Para determinar el grado de conciencia ecológica, se realizó un levantamiento de datos por medio de una encuesta presencial que fue aplicada a 351 personas en Chile, dando como resultado que sólo el $47,86 \%$ de la población tiene conciencia ecológica. Las personas con mayor conciencia ecológica son principalmente personas con edad entre 41 y 64 años, aquellos con un ingreso mensual sobre $\$ 500.000$ pesos y personas que no poseen estudios superiores.

Otras variables que contribuyeron en el cumplimiento del objetivo general fueron la medición de la intención de compra, comportamiento ecológico y conocimiento y credibilidad hacia productos ecológicos. Quienes destacan con una mayor intención de compra son las mujeres, los adultos (entre 41 y 64 años) y personas con ingresos entre $\$ 250.000$ y $\$ 500.000$. Por otro lado, quienes presentan un mayor comportamiento ecológico son las mujeres, las personas con edad entre 41 y 64 años, aquellos que tienen un ingreso mensual sobre $\$ 500.000$ pesos, y las personas 
que no poseen estudios superiores. Finalmente, los consumidores que tienen un mayor conocimiento y credibilidad hacia los productos ecológicos son las mujeres, aquellos con edad entre 28 y 40 años y personas con estudios superiores.

Con el objetivo de determinar la relación directa e indirecta entre las variables conciencia, intención y comportamiento ecológico se utilizó el método de Preacher y Hayes (2008). Los resultados indicaron que el efecto directo entre la conciencia ecológica y el comportamiento ecológico es significativo $(\beta=0,29 ; p<0,01)$, y que el efecto de la conciencia ecológica en el comportamiento ecológico está parcialmente mediado a través de la intención de compra, a un intervalo de confianza del 95\% excluyendo el cero $(0,0610$ a 0,1739$)$.

Cabe destacar que los factores que más inciden en la decisión de compra del consumidor ante este tipo de producto, son la calidad, el precio y la etiqueta del producto ecológico.

Al analizar los resultados y tras la realización de un check list realizado en los supermercados y tiendas de retail del área de análisis, se encontró que existía un gran número de diseños de etiquetas que hacían alusión a una misma característica, provocando confusión y falta de credibilidad en el consumidor. Para solucionar esta problemática se propone diseñar tres etiquetas estándar con las características de producto amigable con el medio ambiente, producto biodegradable y producto hecho con materiales reciclados, respectivamente. Estas etiquetas permitirán resaltar la característica ecológica del producto y disminuir la desconfianza y confusión en el consumidor.

Ante la existencia de diversas características que clasifican a un producto como ecológico, se propone la categorización de los productos por niveles según grado de importancia de las características que presenta, ya sea en su envase, contenido 
y/o proceso de producción. Los niveles de la categorización son independientes y dependerá de cada empresa su modo de aplicación.

Otro problema detectado fue la falta de información en la etiquetas con respecto al beneficio de la inexistencia de ciertos compuestos químicos en los productos. Para hacer frente a este problema se plantea la incorporación de información adicional sobre el beneficio que conlleva la inexistencia de estos compuestos, tanto para la salud de las personas como para el medio ambiente, permitiendo al consumidor adquirir mayor conocimiento acerca de este tipo de características.

Finalmente, se recomienda a las empresas negociar espacios estratégicos en donde se ubiquen sólo productos ecológicos, aumentado el interés del consumidor por la compra de estos.

Con el objetivo de crear o potenciar la conciencia ecológica de los consumidores, se propone la creación de un departamento encargado especialmente de fiscalizar y certificar el eco-etiquetado en Chile, verificando que los productos cumplan con los requisitos necesarios para ser catalogados como ecológicos, evitando engaños que puedan influir negativamente en la decisión de compra del consumidor. Este departamento se encontrará bajo supervisión del Servicio Nacional del Consumidor (SERNAC).

Además, se observó una desinformación por parte de los consumidores, por lo que se propone la realización de eventos ecológicos masivos; donde las empresas den a conocer sus productos con características ecológicas y sus buenas prácticas ambientales, además de informar al consumidor sobre el significado de cada una de las etiquetas existentes en el mercado y las características necesarias que debe tener un producto para ser clasificado en los diferentes niveles. Asimismo, se propone que las empresas le den un giro al objetivo de su publicidad, 
preocupándose de crear una imagen verde real frente a los consumidores. Todo esto con el objetivo de concientizar al consumidor.

A pesar de que en Chile existen empresas que aplican estrategias de marketing ecológico, éstas no son potenciadas, pasando desapercibidas frente a los consumidores. Es por esto, que la información entregada en esta investigación, en conjunto con los planes de acción propuestos, pretenden contribuir al desarrollo de la sustentabilidad de las empresas pertenecientes al área de estudio y aumentar la concientización de la población.

\section{REFERENCIAS}

Bastante, M. J., Capuz, S., Viñoles, R., \& Pacheco, B. (2011). Mercadeo verde, ecoetiquetado y compra responsable. Valencia: Anales de la Universidad Metropolitana.

Bohlen, G., Schlegelmilch, B., \& Diamantopoulus, A. (1993). Measuring ecological concern: A multi-construct perspective. Journal of Marketing Management, 9(4), págs. 415-430.

Calomarde, J. (1995). Práctica perpleja de la Tolerancia. Claves de Razón Práctica(57), 62-65.

CEGESTI (2013). Eco-etiquetas. Costa Rica.

Chamorro, A. (2001). Marketing ecológico; sí, marketing ecológico. Puertas a la Lectura. Universidad de Extremadura.

Comunicación de Sustentabilidad y RSE (2009). expok. Recuperado el 24 de Abril de 2016, de http://www.expoknews.com

Consejería de Medio Ambiente y Ordenación de Territorio (2011). Junta de Andalucia. Recuperado el 24 de Abril de 2016, de http://www.juntadeandalucia.es

D'Souza, C. (2004). Ecolabel programmes: a stakeholder (consumer) perspective. Corporate Communications: An International Journal, 9(3), págs. 179188. 
Dangierico, R., \& Pontrandolfo, P. (2010). From green product definitions and classifications to the Green Option Matrix. Journal of Cleaner Production, 18(16), págs. 1608-1628.

Dunlap, R., \& K.D., V. L. (1978). The new environmental paradigm: A proposed measuring instrument and preliminary results. Journal of Environmental Education, 9, págs. 10-19.

Easterling, D., Miller, S., \& Weinberger, N. (1995). Environmental consumerism: A process of children's socialization and families' resocialization. Psychology \& Marketing, 12(6), págs. 531-550.

Fondo mundial para la Naturaleza (2015). Ecoportal.net. Recuperado el 23 de Abril de 2016, de http://www.ecoportal.net/

Fraj, E., \& Martinez, E. (2002). Comportamiento del consumidor ecológico. ESIC Editorial.

Fundación Universidad Nacional Autónoma de México (2013). Fundación UNAM. Recuperado el 13 de abril de 2016, de Fundación UNAM: fundacionunam.org.mx

Gazulla, C. (2010). Sellos Ambientales Aplicados a Productos. Valladolid, España.

George, D., \& Mallery, P. (1994). SPSS/pc + step by step: A Simple Guide and reference. Belmont : Wadsworth Publishing company.

Grunert, S. C., \& Kristensen, K. (1992). The green consumer: some Danish evidence. Marketing for Europe-Marketing for the Future, 525-39.

Grunert, S., \& Rohme, N. (1992). Consumers'envirnomental concern: Are we really taping true concern taht relates to environmentally ethic behavior? Proceedings of the Esomar Conference Marketing and Reserach Under a New World Order.

Jansson, J., Marell, A., \& Nordlund, A. (2010). Green consumer behavior: determinants of curtailment and eco-innovation adoption. Journal of Consumer Marketing, 27(4), págs. 358-370.

Karp, D. G. (1996). Values and their effect on pro-environmental behavior. Environment and behavior, 28(1), págs. 111-133. 
Kinnear, T., \& Taylor, J. (1973). The effect of ecológical concern on brand perceptions. Journal of Marketing Research, 10(2), págs. 191-197.

Ling-yee, L. (1997). Effect of collectivist orientation and ecological attitude on actual environmental commitment: The moderating role of consumer demographics and product involvement. Journal of International Consumer Marketing, 9(4), págs. 31-53.

Maheswari, D. M. (2015). Purchase Behaviour of Consumers towards Green Products . The Internatinal Journal of Bussines and Management, 147-149.

Oficina de Estudios y Políticas Agrarias (2014). www.odepa.cl. Recuperado el 20 de Abril de 2016, de http://www.odepa.cl

Peattie, K., \& Charter, M. (2003). Green Marketing. En M. Baker, \& M. Baker (Ed.), The Marketing Book (5 ed., Vol. 5, págs. 726-755). Butterworth-Heinemann.

Pedreros, A. M. (2014). La educación ambiental en Chile, una tarea aun pendiente. Ambiente \& Sociedad, 177-198.

Penela, A. C. (2010). Ecoetiquetado de bienes y servicios para un desarrollo sostenible. España: AENOREdiciones.

Polonsky, M. (1994). An Introduction To Green Marketing. Electronic Green Journal, 1(2).

Preacher, K. J., \& Hayes, A. F. (2008). Asymptotic and resampling strategies for assessing and comparing indirect effects in multiple mediator models. Behavior research methods, 40(3), 879-891.

ProArgentina (2005). Productos Ecológicos. Recuperado el 20 de Abril de 2016, de http://www.ebrary.com.ezproxy.ucn.cl

Reinhardt, F. (1998). Evironmental Product Differentation: implication for corporate strategy. California Review Management, 40(4), 43-73.

Senerman, M., \& Aguirre, M. (2015). Fundación Chile. Recuperado el 30 de abril de 2016, de http://www.fch.cl

Servicio Nacional del Consumidor (2012). Informe Publicidad Mediambiental.

Vicente, M. (2014). Marketing ecológico. Oportunidades, políticas y retos. En J. Cubillo, \& A. Blanco, Estrategias de Marketing Sectorial (pág. 268). País Vasco: 
ESIC Editorial.

Webb, D. J., Mohr, L. A., \& Harris, K. E. (2008). A re-examination of socially responsible consumption and its measurement. Journal of Business Research, 61(2), págs. 91-98.

Weigel, R., \& Weigel, J. (1978). Environmental concern the development of a measure. Environmental and Behavior, 10(1), págs. 3-15.

Zumbo, B. D., \& Rupp, A. (2004). Responsible modelling of measurement data for appropriate inferences: Important advances in reliability. (D. Kaplan, Ed.) Thousand Oaks, CA. 